\title{
The Prognostic Impact of Unplanned Excisions in a Cohort of 728 Soft Tissue Sarcoma Patients: A Multicentre Study
}

\author{
Maria Anna Smolle ${ }^{1}$, Per-Ulf Tunn, PD MD ${ }^{2}$, Elisabeth Goldenitsch, MD $^{3}$, Florian Posch, MSc MD ${ }^{4}$, \\ Joanna Szkandera, PD $\mathrm{MD}^{4}$, Marko Bergovec, $\mathrm{MD}^{1}$, Bernadette Liegl-Atzwanger, $\mathrm{MD}^{5}$, and Andreas Leithner, \\ MD $^{1}$
}

${ }^{1}$ Department of Orthopaedic Surgery, Medical University of Graz, Graz, Austria; ${ }^{2}$ Sarcoma Centre HELIOS Klinikum Berlin-Buch, Berlin, Germany; ${ }^{3}$ Orthopaedic Hospital Gersthof, Vienna, Austria; ${ }^{4}$ Division of Clinical Oncology, Medical University of Graz, Graz, Austria; ${ }^{5}$ Institute of Pathology, Medical University of Graz, Graz, Austria

\begin{abstract}
Background. Unplanned excisions (UE) of soft tissue sarcomas (STS) carry a high risk for local recurrence (LR) due to marginal/intralesional resections. However, there are reports about improved prognosis for UE patients who have re-resection compared with patients who undergo planned surgery. The present multicentre study was designed to define characteristics of UE patients and to investigate the impact of UE on subsequent therapy and patient outcomes.

Methods. A total of 728 STS patients (376 males, 352 females; mean age: 58 years) who underwent definite surgery at one of three tumour centres were retrospectively included. Time-to-event analyses were calculated with logrank and Gray's tests, excluding patients with primary metastasis $(n=59)$. A propensity-score (PS) of being in the UE group was estimated, based on differences at baseline between the UE group and non-UE group. An inverse-probability-of-UE weight (IPUEW) was generated and time-to-event analyses calculated after IPUEW weighting.

Results. Before referral, $38.6 \%$ of patients $(n=281)$ had undergone UE. Unplanned excision patients were younger $(p=0.036)$, rather male $(p=0.05)$, and had smaller $(p<0.005)$, superficially located tumours $(p<0.005)$. Plastic reconstructions $(p<0.005)$ and adjuvant radiotherapy $(p=0.041)$ more often were needed at re-
\end{abstract}

(C) The Author(s) 2017. This article is published with open access at Springerlink.com

First Received: 12 October 2016;

Published Online: 20 January 2017

M. A. Smolle

e-mail: maria.smolle@cbmed.at resection. In univariable analysis, re-resected patients had improved overall survival (OS; $p=0.027$ ) and lower risk of distant metastasis (DM; $p=0.002$ ) than primarily resected patients, whereas risk of LR was similar $(p=0.359)$. After weighting for the IPUEW, however, differences in terms of OS $(p=0.459)$ and risk of DM $(p=0.405)$ disappeared.

Conclusions. The present study does not support prior findings of improved outcome for UE patients. Unplanned excisions have a major impact on subsequent therapy, yet they do not seem to affect negatively the long-term oncology outcome.

Diagnosing soft tissue sarcomas among the great number of benign soft tissue lumps and bumps in routine practice is challenging. Soft tissue sarcomas are rare, accounting for only $0.7 \%$ of all malignancies estimated to be diagnosed in 2016 in the United States. ${ }^{1}$ Also, they often are asymptomatic or cause unspecific signs and symptoms. Therefore, STS patients are at risk of delayed or incorrect diagnosis. ${ }^{2}$ Because of these issues, unplanned excisions (UEs) are frequently performed. The proportion of patients referred following UE ranges between 18 and $53.3 \%$ of patients treated for STS at a sarcoma centre. ${ }^{3-5}$

Because UEs are usually the result of primary attempts to remove a lesion that is thought to be benign, they carry a high risk of residual tumour. ${ }^{4,6}$ Following UE, decision for or against re-resection, adjuvant radiotherapy (RTX), chemotherapy (CTX), or watchful waiting at the specialist centre is based on surgical and histological reports, resection margin status, and imaging taken prior to UE. Considering that marginal or even intralesional resection margins significantly increase the likelihood for local 
recurrence (LR), re-resections aiming at removal of residual tumour tissue often are justified. ${ }^{6}$

The combination of primarily inadequate resection, an unavoidable delay from UE to definite surgery, and the peril of residual tumour tissue all suggest that UE patients would do worse compared with initially adequately treated patients. Surprisingly, several study groups reported that UE patients have an identical or even better prognosis than patients who undergo planned surgery. ${ }^{5-7}$ It was concluded that patients with large and high-grade tumours at complicated anatomical sites are far more likely to be referred directly to a specialist centre, thus causing a selection bias. According to Lewis et al., however, even adjustment for these influential factors revealed a prognostic advantage for re-resected patients. ${ }^{6}$ The present multicentre study was designed to elucidate the baseline characteristics of UE cases, their impact on management at the specialist centre, and their potential influence on patient outcomes.

\section{METHODS}

\section{Patients}

We included 728 patients undergoing surgery for STS between 1998 and 2015 at three sarcoma centres (Department of Orthopaedic Surgery, Medical University Graz; Orthopaedic Hospital Gersthof, Vienna; Sarcoma Centre, HELIOS-Klinikum Berlin-Buch). The mean patients' age was 58 years (range 6-96 years; standard deviation (SD): \pm 16.9 years); 352 were female $(48.4 \%)$ and 376 male (51.6\%).

A standard template was used for data collection at each centre. UEs were defined as unintentionally performed resections of a subsequently histologically verified STS. Original tumour size was ascertained from preoperative MRI and/or medical records or from pathology reports following primary surgery at the specialist centre or index surgery performed elsewhere. Tumour grade was recorded according to the FNCLCC system. Staging was done by using the AJCC Cancer Staging Manual from 2010. Patients with metastatic disease $(n=59)$ or unknown metastatic status at time of diagnosis $(n=11)$ were excluded from time-to-event analyses, resulting in 658 eligible patients.

All patients were discussed in multidisciplinary team meetings and underwent primary surgery or re-resection following UE at one of the three sarcoma centres. Need for plastic, vascular, or endoprosthetic reconstruction at definite surgery was documented. Neoadjuvant, adjuvant, and palliative treatments (RTX, CTX) were recorded.

Follow-up was performed until July 2016. Time to last follow-up or death was calculated from definite surgery to last known date (most recent clinical appointment, telephone contact, or record in obituary column). The study was approved by the institutional review boards at the respective centres.

\section{Statistical Analysis}

Statistical analysis was performed using IBM SPSS statistics version 23.0, Microsoft Excel version 15.19.1 and Stata (Windows version 13.0, Stata Corp., Houston, TX). Continuous variables were summarised as means $\pm \mathrm{SD}$, whereas count data were summarised as absolute frequencies with percentages. Associations between two categorical variables, as tumour grade and UE status, were analysed with $\chi^{2}$ tests (expected cell counts $\geq 5$ ) or Fisher's exact tests (expected cell counts $<5$ ). Means between two groups were compared with $t$ tests or in case of heteroscedasticity with Wilcoxon's rank-sum tests. Median survival was estimated with the reverse Kaplan-Meier method by Schemper and Smith. The Kaplan-Meier product-limit estimator was used to calculate survivor functions. Cumulative incidences of LR and distant metastasis (DM) were estimated with competing risk estimators according to Marubini and Valsecchi, treating death-from-any-cause as the competing event of interest. Survivor and cumulative-incidence functions were compared between two or more groups with log-rank and Gray's tests, respectively. Uni- and multivariable modelling of time-to-event outcomes was performed with Coxproportional hazards models for the overall survival (OS) endpoint and Fine \& Gray's proportional subdistribution hazards models for the LR and DM endpoints.

A propensity score (PS), defined as the probability of undergoing UE, was estimated for each individual patient according to characteristics at baseline. ${ }^{8}$ The PS was calculated with a binary logistic regression model, including the following variables: gender, patient's age, anatomical tumour localisation, histologic subtype, tumour depth, size, and tumour grade. Next, an inverse-probability-of-UE weight (IPUEW) was constructed, defined as 1/PS for patients with prior UE and $1 /(1-\mathrm{PS})$ for directly referred patients. Time-to-event analyses for OS, LR, and DM were then weighted using this IPUEW. Sensitivity analyses used a trimmed IPUEW (i.e., the lowest and highest 5\% were removed) that did not materially alter the observed associations.

\section{RESULTS}

Patient characteristics at baseline, tumour-related parameters, definite treatment, and postoperative information are presented in Table 1 . The mean tumour size was 
TABLE 1 Descriptive analysis for all patients and differences depending on prior unplanned excision (UE)

\begin{tabular}{|c|c|c|c|c|c|}
\hline & \multirow{2}{*}{ Total $(n=728)$} & \multicolumn{2}{|c|}{ Unplanned excision } & \multirow[t]{2}{*}{ Missing } & \multirow[t]{2}{*}{$p$ value ( $\chi^{2}$ test $)$} \\
\hline & & No $(n=447)$ & Yes $(n=281)$ & & \\
\hline \multicolumn{6}{|l|}{ Centre } \\
\hline A & 427 & 262 & 165 & 0 & 0.484 \\
\hline $\mathrm{B}$ & 98 & 65 & 33 & & \\
\hline $\mathrm{C}$ & 203 & 120 & 83 & & \\
\hline \multicolumn{6}{|l|}{ Gender } \\
\hline Female & 352 & 229 & 123 & 0 & 0.050 \\
\hline Male & 376 & 218 & 158 & & \\
\hline \multicolumn{6}{|l|}{ Tumour location } \\
\hline Upper limb & 143 & 73 & 70 & 0 & $<\mathbf{0 . 0 0 5}$ \\
\hline Lower limb & 457 & 310 & 147 & & \\
\hline Trunk & 98 & 50 & 48 & & \\
\hline Other & 30 & 14 & 16 & & \\
\hline \multicolumn{6}{|l|}{ Detailed symptoms } \\
\hline No symptoms & 81 & 54 & 27 & 158 & 0.308 \\
\hline Pain & 88 & 60 & 28 & & \\
\hline Increase & 303 & 202 & 101 & & \\
\hline Pain + increase & 96 & 74 & 22 & & \\
\hline \multicolumn{6}{|l|}{ Tumour size $(\mathrm{cm})$} \\
\hline $0-5$ & 228 & 83 & 145 & 99 & $<\mathbf{0 . 0 0 5}$ \\
\hline $5-10$ & 181 & 125 & 56 & & \\
\hline$>10$ & 220 & 183 & 37 & & \\
\hline \multicolumn{6}{|l|}{ Histology } \\
\hline Liposarcoma & 183 & 126 & 57 & 0 & 0.270 \\
\hline Myxofibrosarcoma & 161 & 100 & 61 & & \\
\hline Leiomyosarcoma & 76 & 44 & 32 & & \\
\hline Synovial Sarcoma & 64 & 34 & 30 & & \\
\hline MPNST & 21 & 12 & 9 & & \\
\hline Fibrosarcoma & 6 & 3 & 3 & & \\
\hline Other & 217 & 128 & 89 & & \\
\hline \multicolumn{6}{|l|}{ Depth } \\
\hline Superficial & 230 & 93 & 137 & 6 & $<\mathbf{0 . 0 0 5}$ \\
\hline Deep & 492 & 352 & 140 & & \\
\hline \multicolumn{6}{|l|}{ Grading } \\
\hline G1 & 135 & 88 & 47 & 38 & 0.298 \\
\hline $\mathrm{G} 2$ & 138 & 79 & 59 & & \\
\hline G3 & 417 & 267 & 150 & & \\
\hline \multicolumn{6}{|l|}{ Staging } \\
\hline IA & 35 & 11 & 24 & 38 & $<\mathbf{0 . 0 0 5}$ \\
\hline IB & 98 & 76 & 22 & & \\
\hline IIA & 138 & 50 & 88 & & \\
\hline IIB & 84 & 59 & 25 & & \\
\hline III & 276 & 198 & 78 & & \\
\hline IV & 59 & 40 & 19 & & \\
\hline \multicolumn{6}{|l|}{ Primary LN metastasis } \\
\hline No & 695 & 430 & 265 & 9 & 0.950 \\
\hline Yes & 24 & 15 & 9 & & \\
\hline
\end{tabular}


TABLE 1 continued

\begin{tabular}{|c|c|c|c|c|c|}
\hline & \multirow[t]{2}{*}{ Total $(n=728)$} & \multicolumn{2}{|c|}{ Unplanned excision } & \multirow[t]{2}{*}{ Missing } & \multirow[t]{2}{*}{$p$ value ( $\chi^{2}$ test) } \\
\hline & & No $(n=447)$ & Yes $(n=281)$ & & \\
\hline \multicolumn{6}{|c|}{ Primary distant metastasis } \\
\hline No & 680 & 412 & 268 & 5 & 0.071 \\
\hline Yes & 43 & 32 & 11 & & \\
\hline \multicolumn{6}{|c|}{ Duration of symptoms (mo) } \\
\hline$<6$ & 315 & 227 & 88 & 175 & 0.008 \\
\hline$>6$ & 238 & 136 & 92 & & \\
\hline \multicolumn{6}{|c|}{ Amputation } \\
\hline No & 663 & 399 & 264 & 0 & 0.031 \\
\hline Yes & 65 & 48 & 17 & & \\
\hline \multicolumn{6}{|c|}{ Plastic reconstruction } \\
\hline No & 505 & 345 & 160 & 3 & $<0.005$ \\
\hline Yes & 220 & 101 & 119 & & \\
\hline \multicolumn{6}{|c|}{ Vascular reconstruction } \\
\hline No & 687 & 419 & 268 & 3 & 0.215 \\
\hline Yes & 38 & 27 & 11 & & \\
\hline \multicolumn{6}{|c|}{ Endoprosthetic devices } \\
\hline No & 677 & 409 & 268 & 3 & 0.022 \\
\hline Yes & 48 & 37 & 11 & & \\
\hline \multicolumn{6}{|c|}{ R-classification } \\
\hline R0 & 583 & 345 & 238 & 71 & 0.001 \\
\hline $\mathrm{R} 1$ & 67 & 52 & 15 & & \\
\hline $\mathrm{R} 2$ & 7 & 7 & 0 & & \\
\hline \multicolumn{6}{|c|}{ Postoperative complications } \\
\hline No & 561 & 347 & 214 & 3 & 0.730 \\
\hline Yes & 164 & 99 & 65 & & \\
\hline \multicolumn{6}{|c|}{ Radiotherapy* } \\
\hline No & 284 & 184 & 100 & 10 & 0.119 \\
\hline Yes & 434 & 256 & 178 & & \\
\hline \multicolumn{6}{|c|}{ Adjuvant radiotherapy } \\
\hline No & 330 & 216 & 114 & 0 & 0.041 \\
\hline Yes & 398 & 231 & 167 & & \\
\hline \multicolumn{6}{|c|}{ Chemotherapy* } \\
\hline No & 501 & 292 & 209 & 10 & 0.009 \\
\hline Yes & 217 & 149 & 68 & & \\
\hline \multicolumn{6}{|c|}{ Local recurrence } \\
\hline No & 618 & 384 & 234 & 5 & 0.431 \\
\hline Yes & 105 & 61 & 44 & & \\
\hline \multicolumn{6}{|c|}{ Distant metastasis } \\
\hline No & 549 & 324 & 225 & 8 & 0.019 \\
\hline Yes & 171 & 118 & 53 & & \\
\hline
\end{tabular}

Statistically significant results are given in bold

* Comprising neoadjuvant, adjuvant, palliative, or combined treatment regimes

$8.9 \mathrm{~cm}$ (range $0.5-47.0 \mathrm{~cm}$; SD: $\pm 6.3 \mathrm{~cm}$ ), being significantly smaller in superficial STS than in tumours underneath the fascia $(6.1 \pm 4.8 \mathrm{~cm} ; 10.2 \pm 6.4 \mathrm{~cm} ; t$ test, $p=0.005)$. With 457 cases, most STS were located in the lower limbs (62.8\%), mainly the thigh. A total of 276 patients had AJCC stage III tumours (40\%), and a further 
59 had evidence of metastatic disease at time of diagnosis $(8.6 \%)$.

The median duration of symptoms before admission was 6 months (range 3 weeks-30 years). In total, 399 patients had noticed a recent increase of the tumour $(70.2 \%)$, which was painless in 303 cases and associated with pain in 96 . An additional 88 patients had experienced pain only $(15.5 \%)$, whereas 81 reported no symptoms at all $(14.3 \%)$.

Altogether, 281 STS patients had undergone UE before referral $(38.6 \%)$, with a similar incidence at each centre (Table 1). The median delay from UE to definite surgery was 7.9 weeks, with $68.7 \%$ of patients treated within 12 weeks $(n=193)$.

At the centres, most patients underwent wide ( $n=492$; $67.6 \%)$ and compartmental tumour resection $(n=85$; $11.7 \%$ ). Limb-salvage surgery was not feasible in $10.7 \%$ of primary surgeries $(n=48)$ compared with $6.0 \%$ of re-resections $(n=17 ; p=0.031)$.

A total of 220 patients required plastic reconstructions, as muscular flaps (35.9\%) and split-skin grafts $(32.3 \%)$. These reconstructions were necessary in $42.7 \%$ of re-resections $(n=119)$ compared with $22.6 \%$ of planned surgeries $(n=101 ; p<0.005)$. At definite surgery, clear tumour margins (R-Classification; R0) could be achieved in 583 cases $(88.7 \%)$, microscopically intralesional margins (R1) in 67 cases (10.2\%), and macroscopically intralesional margins (R2) in 7 cases $(1.1 \%)^{9,10}$ Postoperative complications that necessitated revision developed in 164 patients $(22.6 \%$; Table 1$)$. The rate of postoperative complications did not significantly differ between UE and non-UE -patients (23.3 vs. $22.1 \%$; $p=0.730$ ).

Of 434 patients receiving RTX $(60.4 \%)$, the vast majority underwent postoperative irradiation of the tumour bed $(n=398)$. Patients who underwent re-resection received adjuvant RTX more often than patients who had planned surgery (59.4 vs. $51.7 \% ; p=0.041$ ).

Altogether, $30.2 \%$ of patients $(n=217)$ were administered CTX in a neoadjuvant $(n=45)$, adjuvant $(n=70)$, palliative $(n=33)$, or combined treatment regimen $(n=69)$. After a median follow-up of 5.5 years (25th75 th percentile $3.0-8.4$ years), 82 patients were alive with disease $(11.3 \%)$ and 399 had no evidence of disease (54.8\%); 129 patients died of STS (17.7\%) and 73 died due to other causes $(10 \%)$. UE patients accounted for $31 \%$ $(n=40)$ and non-UE patients for $69.0 \%(n=89)$ of cancer-related deaths.

Of 658 patients with localised disease at time of diagnosis, 92 subsequently developed LR (14.0\%), whereas 137 patients (20.8\%) developed DM. The 5- and 10-year OS rates for all patients were 77.7 and $63.8 \%$, respectively. 5 and 10-year risks of LR were estimated at 12.8 and $20.4 \%$ and corresponding risks of DM at 22.3 and $25.0 \%$ (Fig. 1). Tumour size $(p=0.004)$, grade $(p=0.013)$, stage

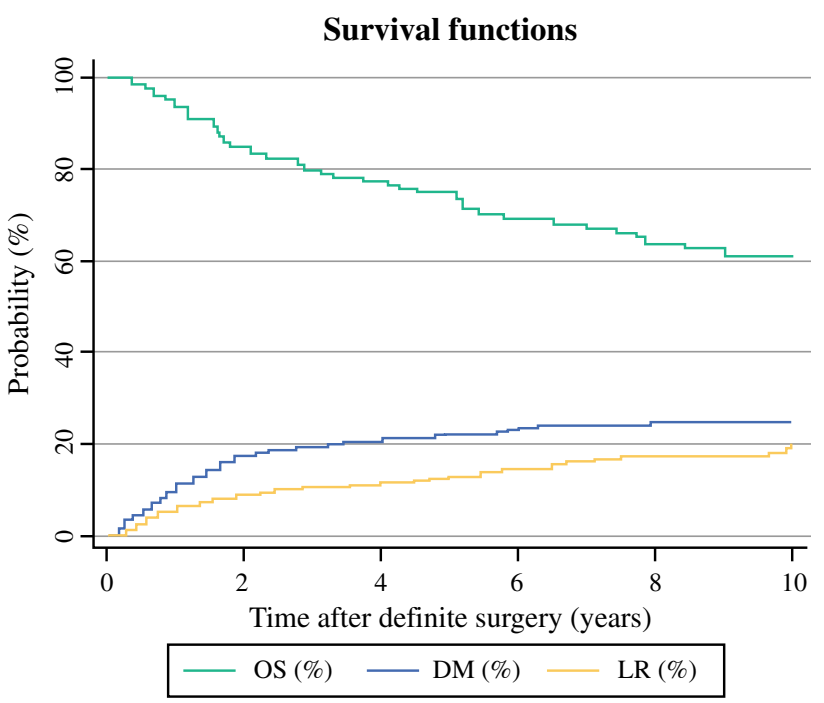

FIG. 1 Overall survival (OS) and cumulative incidences of local recurrence (LR) as well as distant metastasis (DM) calculated for patients with localised disease at time of diagnosis $(n=658)$

$(p<0.005)$, histological subtype $(p=0.007)$, symptoms lasting for less than 6 months $(p<0.005)$, and use of CTX $(p<0.005)$ were significant predictors of survival using univariable analysis (Table 2). UE patients undergoing definite surgery later than 12 weeks had a $113 \%$ higher relative risk for LR (subdistribution hazard ratio (SHR): 2.135; 95\% confidence interval (CI) 1.108-4.112; $p=0.023$ ) and a $186 \%$ higher relative risk of DM (SHR: $2.863 ; 95 \%$ CI $1.483-5.537 ; p=0.002)$ than those treated earlier than 12 weeks.

Univariable analysis revealed that UEs with consecutive re-resection were significantly associated with improved OS compared with primary surgeries (hazard ratio (HR): 0.689; 95\% CI 0.494-0.961; $p=0.027$; Fig. 2a). At 5 and 10 years, 82.3 and $67.5 \%$ of patients with underwent resection were alive compared with 74.8 and $61.4 \%$ of patients after planned surgery. UE patients developed DM less frequently than patients with planned surgery ( $p=0.002$; Fig. 2b), whereas LR-free survival rates were similar for both groups ( $p=0.359$; Fig. 2c).

In multivariable analysis, tumour size and grade were confirmed as independent prognostic factors for OS, whereas the favourable "effect" of UE did not prevail after adjustment for selected covariates (HR: 0.737; 95\% CI 0.501-1.085; $p=0.122$; Table 3).

However, some significant differences at baseline between patients undergoing UE and those with planned surgery were observed (Table 1). Tumours undergoing UE were significantly smaller $(p<0.005)$, were preferably located superficially ( $p<0.005$ ), and more often had been noticed by patients some time ago $(p=0.008)$. Male patients were more likely to have their tumours excised 
TABLE 2 Univariable Cox-regression analysis regarding overall survival for patients without primary metastasis at time of diagnosis

\begin{tabular}{|c|c|c|c|c|}
\hline & \multirow[t]{2}{*}{ HR } & \multicolumn{2}{|c|}{$95 \% \mathrm{CI}$} & \multirow[t]{2}{*}{$p$ value } \\
\hline & & Lower & Upper & \\
\hline \multicolumn{5}{|l|}{ Gender $(n=658)$} \\
\hline Female & 1 & & & 0.794 \\
\hline Male & 1.043 & 0.762 & 1.427 & \\
\hline \multicolumn{5}{|c|}{ Tumour location $(n=658)$} \\
\hline Upper limb & 1 & & & \\
\hline Lower limb & 0.788 & 0.363 & 1.711 & 0.547 \\
\hline Trunk & 0.774 & 0.376 & 1.592 & 0.486 \\
\hline Other & 0.712 & 0.301 & 1.683 & 0.439 \\
\hline \multicolumn{5}{|c|}{ Tumour size $(\mathrm{cm})(n=568)$} \\
\hline $0-5$ & 1 & & & \\
\hline $5-10$ & 1.953 & 1.250 & 3.051 & 0.003 \\
\hline$>10$ & 1.953 & 1.276 & 2.987 & 0.002 \\
\hline \multicolumn{5}{|l|}{ Histology $(n=652)$} \\
\hline Fibrosarcoma & $\mathrm{NE}$ & $\mathrm{NE}$ & $\mathrm{NE}$ & NE \\
\hline Liposarcoma & 1 & & & \\
\hline Myxofibrosarcoma & 2.081 & 1.225 & 3.536 & 0.007 \\
\hline Leiomyosarcoma & 3.510 & 1.966 & 6.266 & $<\mathbf{0 . 0 0 5}$ \\
\hline Synovial sarcoma & 2.909 & 1.548 & 5.466 & 0.001 \\
\hline MPNST & 3.411 & 1.522 & 7.648 & 0.003 \\
\hline Other & 3.212 & 1.951 & 5.289 & $<\mathbf{0 . 0 0 5}$ \\
\hline \multicolumn{5}{|l|}{ Depth $(n=652)$} \\
\hline Superficial & 1 & & & 0.762 \\
\hline Deep & 1.055 & 0.747 & 1.490 & \\
\hline \multicolumn{5}{|l|}{ Grading $(n=625)$} \\
\hline G1 & 1 & & & \\
\hline G2 & 2.526 & 1.218 & 5.239 & 0.013 \\
\hline G3 & 5.451 & 2.854 & 10.411 & $<\mathbf{0 . 0 0 5}$ \\
\hline \multicolumn{5}{|l|}{ Staging $(n=623)$} \\
\hline IA / IB & 1 & & & \\
\hline IIA / IIB & 2.846 & 1.434 & 5.648 & 0.003 \\
\hline III & 6.170 & 3.214 & 11.843 & $<\mathbf{0 . 0 0 5}$ \\
\hline \multicolumn{5}{|c|}{ Unplanned excision $(n=658)$} \\
\hline No & 1 & & & $\mathbf{0 . 0 2 7}$ \\
\hline Yes & 0.689 & 0.494 & 0.961 & \\
\hline \multicolumn{5}{|c|}{ Duration of symptoms $(\mathrm{mo})(n=492)$} \\
\hline$<6$ & 1 & & & $<\mathbf{0 . 0 0 5}$ \\
\hline$>6$ & 0.538 & 0.369 & 0.782 & \\
\hline \multicolumn{5}{|c|}{ Postoperative complications $(n=654)$} \\
\hline No & 1 & & & 0.870 \\
\hline Yes & 1.030 & 0.718 & 1.478 & \\
\hline \multicolumn{5}{|c|}{ Radiotherapy* $(n=651)$} \\
\hline No & 1 & & & 0.400 \\
\hline Yes & 1.152 & 0.829 & 1.600 & \\
\hline \multicolumn{5}{|c|}{ Adjuvant radiotherapy $(n=657)$} \\
\hline No & 1 & & & 0.495 \\
\hline Yes & 1.118 & 0.812 & 1.539 & \\
\hline
\end{tabular}


TABLE 2 continued

\begin{tabular}{llll}
\hline & HR & \multicolumn{9}{c}{$p$ CI value } \\
\cline { 3 - 4 } & & Lower & Upper \\
Chemotherapy* $(n=651)$ & 1 & & $<\mathbf{0 . 0 0 5}$ \\
No & 1.893 & 1.378 & 2.601 \\
\hline
\end{tabular}

Statistically significant results are given in bold

$N E$ nonestimable: hazard ratio could not be estimated for the fibrosarcoma-subgroup ( $n=6$, no deaths during follow-up period)

* Comprising neoadjuvant, adjuvant, palliative, or combined treatment regimes

inadvertently compared with females $(p=0.05)$. UE patients were younger on average than directly referred ones ( 56.5 vs. 59.2 years; $p=0.036$ ). Because most of the abovementioned parameters are well-established prognostic factors, they potentially have a greater influence on survival than UE itself.

To adjust for these confounding factors, IPUEW analysis was performed as described in the "Methods" section. The odds of being in the UE group differed according to whether tumours were superficially located (odds ratio (OR) for superficial location to being a non-UE patient: $0.29, p<0.0001)$. After adjusting for the PS, this difference vanished (OR: $1.04, p=0.875$ ), thus supporting the concept that this PS adequately controlled for imbalances between UE- and non-UE patients. After weighting for the IPUEW score, UEs with subsequent re-resection were not significantly associated with an improved survival any more (HR: 0.85 ; 95\% CI $0.56-1.30 ; p=0.459$; Fig. 2d), in conformity with the multivariable analysis. Differences in terms of DM-free survival were likewise lost following IPUEW score weighting ( $p=0.405$; Fig. 2e).

\section{DISCUSSION}

In this retrospective, multicentre study, we analysed the association between unplanned excision, subsequent therapy, local recurrence, distant metastasis, and overall survival in 728 patients with STS. In univariable analysis, patients with prior UE had significantly better overall survival than patients with primary surgery at the tumour centre. However, favourable prognostic factors, such as small, low-grade tumours and superficial location, were more common in patients with UE. These data strongly support the concept that UEs per se, given that they are followed by appropriate definite surgery at a tertiary centre, have no major prognostic impact in patients with STS.

Patients referred following UEs are a widely known phenomenon, accounting for up to $53.3 \%$ of all STS patients treated at tumour centres. ${ }^{3-5}$ In our cohort, $38.6 \%$ of patients treated at one of the three centres had undergone UE outwards. The rates that we found are comparable to the $37 \%$ reported by Lewis et al. and $34.8 \%$ observed by Koulaxouzidis et al. ${ }^{6,11}$

A combination of various factors tempts physicians to excise a soft tissue tumour. STS from our cohort undergoing UE had been small and were preferably located clearly visible in the subcutis. Similar observations have been made by other investigators. ${ }^{3,6,12}$ Interestingly, the quality of symptoms did not significantly alter the rate of inadvertent resections, albeit patients reporting a long history of complaints were more likely to undergo UE. Moreover, we observed a difference for gender and age; significantly more males and younger patients underwent UE.

Due to smaller and more often superficially located tumours, UE patients were less likely to undergo amputation at definite surgery compared with directly referred patients. However, in $42.7 \%$ of re-resections after UE, muscular flaps and split-skin grafts were required, whereas plastic reconstructions became necessary in $22.6 \%$ of primary surgeries only. Similar disparities have been reported by Potter et al. In their cohort, however, only $5 \%$ of planned surgeries but $30 \%$ of re-resections necessitated plastic-reconstructive soft tissue coverage. ${ }^{13}$

Because of soft tissue damage, unclear resection margins or frank residual tumour following UE, radical therapeutic approaches are chosen during re-resection in relation to the original tumour size. Whether irradiation following re-resection reduces rate of LR has been discussed controversially. Most authors have concluded that RTX should be administered deliberately, based on tumour extent, postoperative margin status, and histology. ${ }^{13-15}$ At least in high-grade STS $>5 \mathrm{~cm}$ with a deep location, the risk for LR seems to be reduced by RTX. ${ }^{14}$ The small average-tumour size notwithstanding, significantly more of our UE patients $(59.4 \%)$ underwent postoperative irradiation compared with patients who had planned surgery $(51.7 \%)$. These findings are consistent with previous observations. $^{13}$

Despite more often requiring plastic reconstructions and receiving adjuvant RTX, UE patients did not develop postoperative complications more frequently than non-UE 


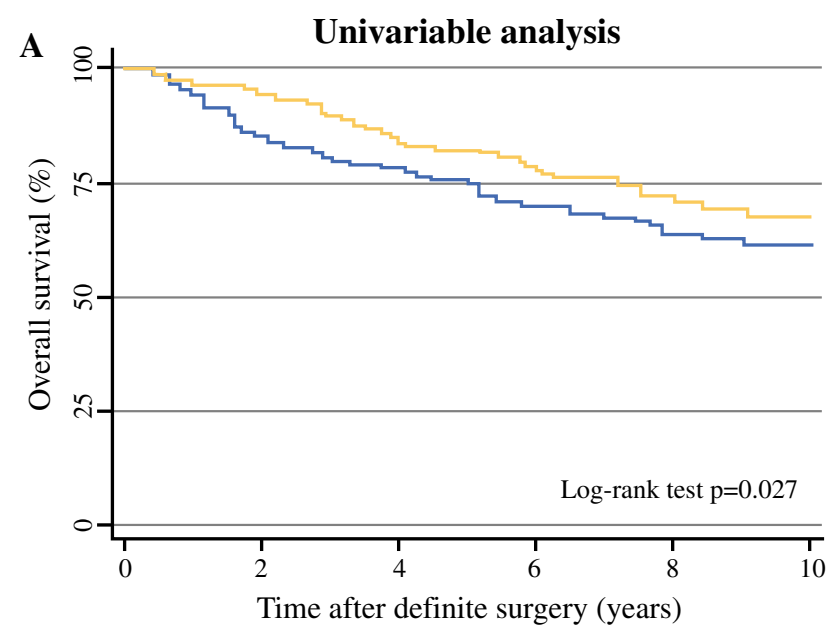

No UE $(n=403) \longrightarrow$ UE $(n=255)$

B

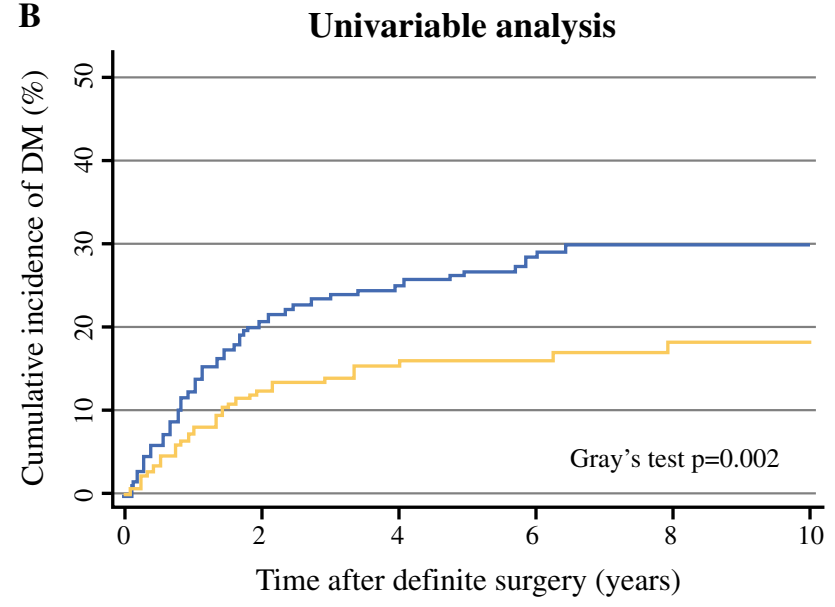

$\longrightarrow$ No UE $(n=388) \longrightarrow$ UE $(n=246)$

C

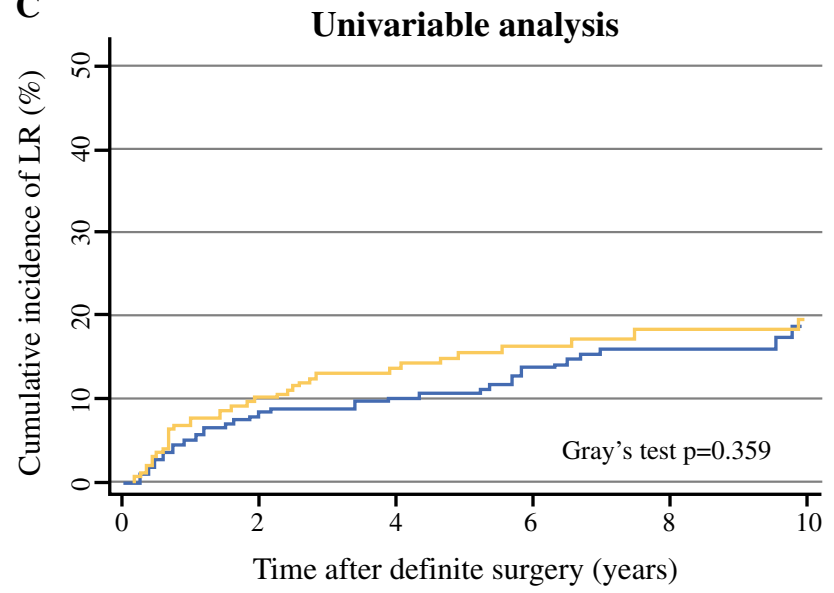

No UE $(n=304) \longrightarrow$ UE $(n=166)$

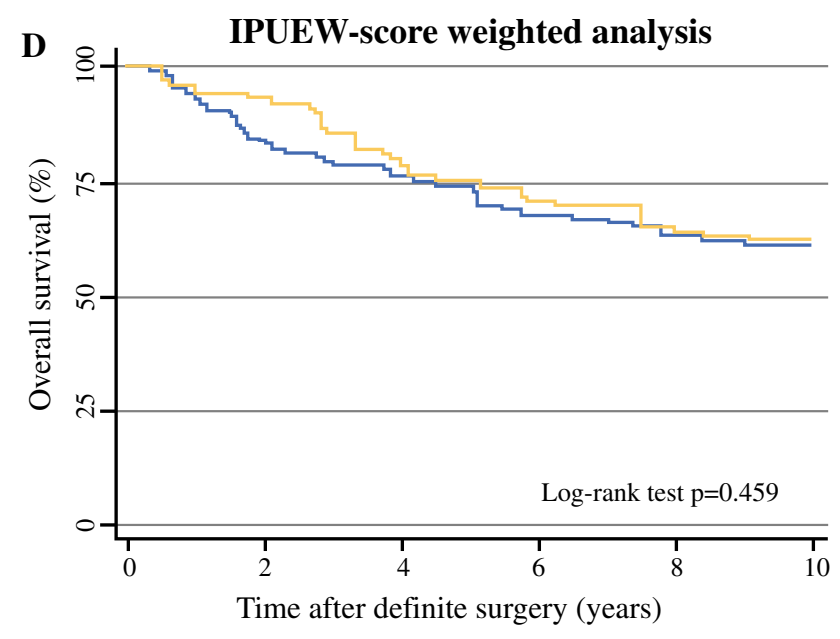

No UE $(n=403) \quad$ UE $(n=255)$

IPUEW-score weighted analysis

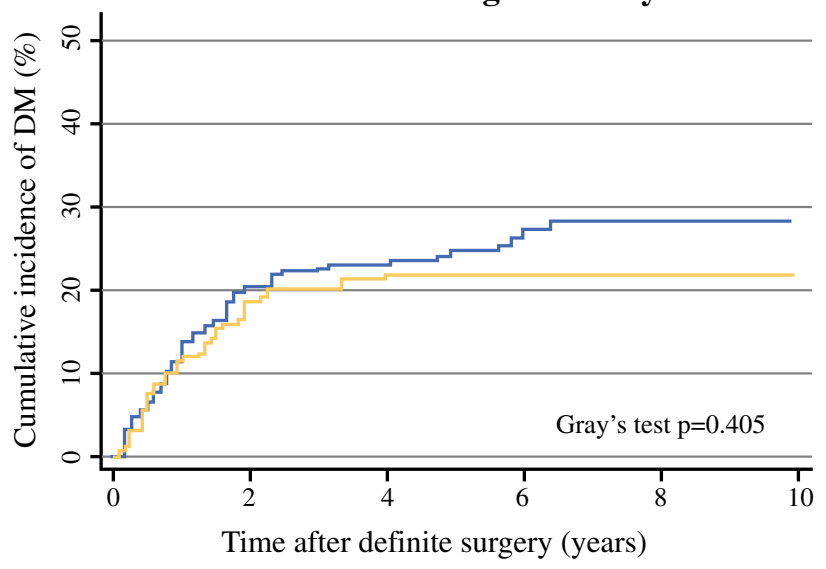

$\longrightarrow$ No UE $(n=388) \longrightarrow \operatorname{UE}(n=246)$

F IPUEW-score weighted analysis

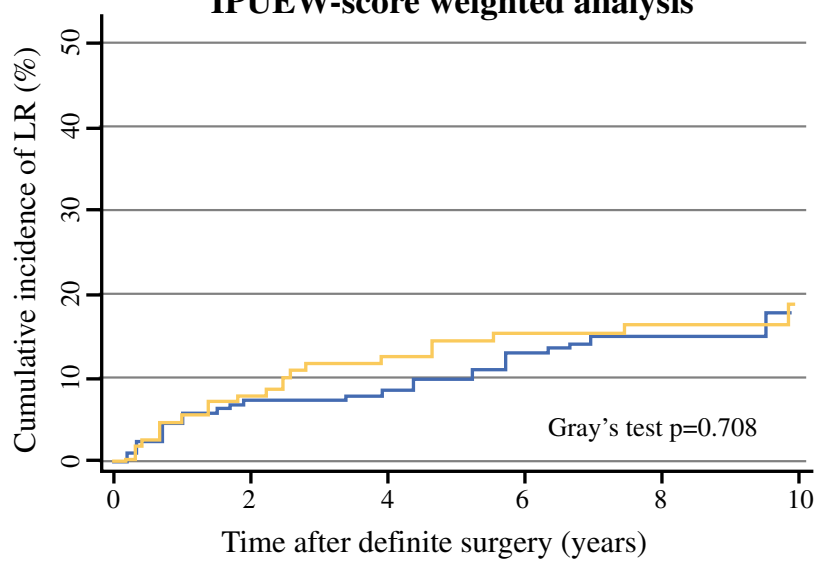


4FIG. 2 Comparison between patients with prior unplanned excision (UE) and patients undergoing planned surgery (no UE). Juxtaposition of results obtained by univariable analysis and IPUEW-weighted analysis in terms of overall survival ( 2 a vs. $2 \mathbf{d}$ ), risk of local recurrence (2c vs. 2f; LR analysis using a $5 \%$ trimmed IPUEW), and risk of distant metastasis (2b vs. $2 \mathbf{e})$

patients in our cohort. There is no doubt, however, that many UE-patients could be protected from disproportionate treatment strategies in relation to the underlying pathology if they would be referred directly.

Preoperative RTX has proven effective in the management of distinct STS subtypes. ${ }^{16}$ However, administration of RTX before definite surgery may not be suitable for UE patients, due to an elevated risk of perioperative complications. $^{17}$

As a result of more complex treatment approaches, UE are associated with considerable financial consequences. According to Alamanda et al., professional costs (i.e., charges billed by surgeons) significantly increased in reresections compared with primary surgeries. ${ }^{18}$ Even after adjustment for influential parameters, such as tumour grade, site, size, and the fitness of patients before surgery, re-excisions were associated with a $33 \%$ increase in professional charges. $^{18}$

Patients subjected to definite surgery at one of the three tumour centres later than 12 weeks following UE had a higher risk for LR and DM compared with those undergoing re-resection shortly after inadvertent resection. Comparable results have been reported by Funovics et al. analysing 310 STS patients with a history of prior UE. $^{19}$
The OS rate of our patients with localised disease at time of diagnosis was $77.7 \%$ at 5 years and $63.8 \%$ at 10 years. In line with literature, large tumour size and high tumour grade were independent negative prognostic factors. ${ }^{15}$ Several studies have reported an elevated risk for LR despite wide re-resection for prior inadvertently excised STS compared with patients who underwent planned surgery, whereas others did not observe any difference. $5,6,13,20,21$

We found that patients who underwent UE with consecutive re-resection had a significantly better prognosis in the univariable analysis $(p=0.027)$. Moreover, they seemed to have a lower risk of developing DM, whereas LR-free survival was comparable to patients treated adequately from the beginning.

After adjusting for the IPUEW score, however, the differences in DM-free survival $(p=0.405)$ and OS $(p=0.459)$ between patients with and without prior UE disappeared. Fiore et al. reported the same conjuncture in a cohort of 597 patients with STS, of whom $53.3 \%$ had been referred after UE. ${ }^{5}$ They used a multivariate analysis, including tumour size, depth, grade, and histological subtype, to estimate the effect of UE on OS. ${ }^{5}$ In this study, we also applied multivariable analysis but additionally used IPUEW score-weighting. Both the multivariable adjustment and the IPUEW score-weighted analysis supported our conclusions.

A limitation of this study is its retrospective design, entailing dependency on exhaustive patient history and detailed medical records during follow-up. Additionally, our cohort comprises likewise patients with STS of the

TABLE 3 Multivariable Cox-regression analysis concerning overall survival for patients without primary metastasis at time of diagnosis $(n=543)$

\begin{tabular}{|c|c|c|c|c|}
\hline & \multirow[t]{2}{*}{ HR } & \multicolumn{2}{|l|}{$95 \% \mathrm{CI}$} & \multirow[t]{2}{*}{$p$ value } \\
\hline & & Lower limit & Upper limit & \\
\hline \multicolumn{5}{|c|}{ Tumour size $(\mathrm{cm})$} \\
\hline $0-5$ & 1 & & & \\
\hline $5-10$ & 1.864 & 1.168 & 2.975 & 0.009 \\
\hline$>10$ & 2.309 & 1.438 & 3.707 & 0.001 \\
\hline \multicolumn{5}{|l|}{ Depth } \\
\hline Superficial & 1 & & & 0.266 \\
\hline Deep & 0.793 & 0.527 & 1.193 & \\
\hline \multicolumn{5}{|l|}{ Grading } \\
\hline G1 & 1 & & & \\
\hline $\mathrm{G} 2$ & 2.809 & 1.337 & 5.902 & 0.006 \\
\hline G3 & 5.965 & 3.066 & 11.532 & $<0.005$ \\
\hline \multicolumn{5}{|c|}{ Unplanned excision } \\
\hline No & 1 & & & 0.122 \\
\hline Yes & 0.737 & 0.501 & 1.085 & \\
\hline
\end{tabular}

Statistically significant results are given in bold 
extremities, trunk, head, and neck. Moreover, it hardly is possible to assess the impact of potential overtreatment following UE on the patient's prognosis in a retrospective setting and results apply only to UE patients who have actually been referred to a tertiary centre. Thus, UE patients may have a significantly worse prognosis in case treatment based on the same parameters used for directly referred patients (e.g., tumour size, depth, grade).

\section{CONCLUSIONS}

Our study does not support prior findings that unplanned excisions improve patient outcomes. There also is no evidence that inadvertent resections might be harmful to prognosis. Unplanned excisions have, however, an influence on the extent of subsequent therapeutic procedures. More radical surgical approaches requiring plastic reconstruction and the increased need for postoperative radiotherapy compromise the medical condition of patients with prior unplanned excisions. For that reason, they must be avoided by all means.

ACKNOWLEDGEMENTS Open access funding provided by Medical University of Graz.

OPEN ACCESS This article is distributed under the terms of the Creative Commons Attribution 4.0 International License (http:// creativecommons.org/licenses/by/4.0/), which permits unrestricted use, distribution, and reproduction in any medium, provided you give appropriate credit to the original author(s) and the source, provide a link to the Creative Commons license, and indicate if changes were made.

\section{REFERENCES}

1. Siegel RL, Miller KD, Jemal A. Cancer statistics, 2016. $C A$ Cancer J Clin. 2016;66:7-30.

2. Springfield DS, Rosenberg A. Biopsy: complicated and risky. $J$ Bone Joint Surg Am. 1996;78(5):639-43.

3. Chandrasekar CR, Wafa H, Grimer RJ, Carter SR, Tillman RM, Abudu A. The effect of an unplanned excision of a soft-tissue sarcoma on prognosis. J Bone Joint Surg Br. 2008;90(2):203-8.

4. Noria S, Davis A, Kandel R, et al. Residual disease following unplanned excision of soft-tissue sarcoma of an extremity. $J$ Bone Joint Surg Am. 1996;78(5):650-5.

5. Fiore M, Casali PG, Miceli R, et al. Prognostic effect of reexcision in adult soft tissue sarcoma of the extremity. Ann Surg Oncol. 2006;13(1):110-7.

6. Lewis JJ, Leung D, Espat J, Woodruff JM, Brennan MF. Effect of reresection in extremity soft tissue sarcoma. Ann Surg. 2000;231(5):655-63.
7. Morii $\mathrm{T}$, Aoyagi $\mathrm{T}$, Tajima $\mathrm{T}$, Yoshiyama A, Ichimura S, Mochizuki K. Unplanned resection of a soft tissue sarcoma: clinical characteristics and impact on oncological and functional outcomes. J Orthop Sci. 2015;20(2):373-9.

8. Rosenbaum PR, Rubin DB. The central role of the propensity score in observational studies for causal effects. Biometrika. 1983;70(1):41-55.

9. Tunn PU, Kettelhack C, Durr HR. Standardized approach to the treatment of adult soft tissue sarcoma of the extremities. Recent Results Cancer Res. 2009;179:211-28.

10. Kainhofer V, Smolle MA, Szkandera J, et al. The width of resection margins influences local recurrence in soft tissue sarcoma patients. Eur J Surg Oncol. 2016;42(6):899-906.

11. Koulaxouzidis G, Schwarzkopf E, Bannasch H, Stark GB. Is revisional surgery mandatory when an unexpected sarcoma diagnosis is made following primary surgery? World J Surg Oncol. 2015;13:306.

12. Dyrop HB, Safwat A, Vedsted P, et al. Characteristics of 64 sarcoma patients referred to a sarcoma center after unplanned excision. J Surg Oncol. 2016;113(2):235-9.

13. Potter BK, Adams SC, Pitcher JD, Jr., Temple HT. Local recurrence of disease after unplanned excisions of high-grade soft tissue sarcomas. Clin Orthop Relat Res. 2008;466(12):3093-100.

14. Casali PG, Blay JY, experts ECECPo. Soft tissue sarcomas: ESMO Clinical Practice Guidelines for diagnosis, treatment and follow-up. Ann Oncol. 2010;21 Suppl 5:v198-v203.

15. Pisters PW, Leung DH, Woodruff J, Shi W, Brennan MF. Analysis of prognostic factors in 1,041 patients with localized soft tissue sarcomas of the extremities. J Clin Oncol. 1996;14(5):1679-89.

16. Levy A, Bonvalot S, Bellefqih $\mathrm{S}$, et al. Is preoperative radiotherapy suitable for all patients with primary soft tissue sarcoma of the limbs? Eur J Surg Oncol. 2014;40(12):1648-54.

17. Jones DA, Shideman C, Yuan J, et al. Management of unplanned excision for soft-tissue sarcoma with preoperative radiotherapy followed by definitive resection. Am J Clin Oncol. 2016;39(6):586-92.

18. Alamanda VK, Delisca GO, Mathis SL, et al. The financial burden of reexcising incompletely excised soft tissue sarcomas: a cost analysis. Ann Surg Oncol. 2013;20(9):2808-14.

19. Funovics PT, Vaselic S, Panotopoulos J, Kotz RI, Dominkus M. The impact of re-excision of inadequately resected soft tissue sarcomas on surgical therapy, results, and prognosis: a single institution experience with 682 patients. J Surg Oncol. 2010;102(6):626-33.

20. Qureshi YA, Huddy JR, Miller JD, Strauss DC, Thomas JM, Hayes AJ. Unplanned excision of soft tissue sarcoma results in increased rates of local recurrence despite full further oncological treatment. Ann Surg Oncol. 2012;19(3):871-7.

21. Zagars GK, Ballo MT, Pisters PW, Pollock RE, Patel SR, Benjamin RS. Surgical margins and reresection in the management of patients with soft tissue sarcoma using conservative surgery and radiation therapy. Cancer. 2003;97(10):2544-53. 